Modelling the Effect of Treatment and Behavioral Change

in HIV Transmission Dynamics

Ying-Hen Hsieh

and

Jorge X. Velasco-Hernandez

BU-1143-M

December 1991 
MODELLING THE EFFECT OF TREATMENT AND BEHAVIORAL CHANGE IN HIV TRANSMISSION DYNAMICS.

\author{
Ying-Hen Hsieh \\ Depatment of Applied Mathematics \\ National Chung-Hsing University, Taichung, Taiwan, R0C \\ and \\ Jorge X. Velasco-Hernandez \\ Biometrics Unit 322 Warren Hall, \\ Cornell University Ithaca NY 14853; \\ Departamento El Hombre y su Ambiente \\ Universidad Autonoma Metropolitana-Xochimilco, Mexico City.
}




\title{
YODELLING THE EFFECT OF TREATMENT AND BEHAVIORAL CHANGE IN HIV TRANSMISSION DYNAMICS.
}

\begin{abstract}
In this paper we analyze a model for the HIV-infection transmission in a male homosexual cohort. In the model we consider two types of infected individuals. Those that are infected but are not under any sort of clinical/therapeutical treatment and those who are. The two groups of infectives differ in their incubation time, contacts with the susceptible individuals, and probability of transmission. The analytical results show that change in sexual behavior is important in lowering prevalence and incidence rate and, eventually, in driving the population toward the disease-free equilibrium.
\end{abstract}




\section{Introduction.}

The most recent figure on the spread of a world-wide AIDS epidemic shows over 10 million individuals infected with HIV virus. In United States alone, 117,781 cases of AIDS were reported by CDC as of December 1989 with over a million more individuals thought to have been infected with HIV. The dread devastation of an AIDS epidemic predicted by many seems to have arrived only a short ten years after AIDS was first recognized as a new form of sexually transmitted disease (Masur et al. [1981]). Despite major public health efforts, the research for an antiviral drug with a direct effect against the HIV virus has yet to bear fruit. However, several drugs have been shown to work against HIV and its related illness, of which only two have been approved by the U. S. Food and Drug Administration for use in therapeutic treatment for AIDS: AZT (azidothymidine) and ddI. Although AZT has been demonstrated to prolong the survival time of AIDS patients as early as 1987 (Fischl et al.), its use in therapy can produce several severe side effects including bone marrow suppression, anemia, and liver problems (see e.g., Broder and Fauci [1988], Dournon et al. [1988], or Fisch1 et al. [1989]). 0ther side effects such as sleep disburbances have also been reported in AZT treatment of patients (Richman et al. [1987]). While ddI works against Pneumocystis carinii pneumonia (PCP), a leading cause of death among AIDS patients, and is less toxic than AZT.

To aliviate the side effects of AZT, treatment with AZT is alternated with other drugs or procedures that have been found to reduce the side effects, while techniques such as liposomal encapsulation of AZT have been 
reported to decrease bone marrow toxicity (Phillips et al. [1991]). Presently, the treatment program of an AIDS patient must take into account the patient's overall health, the presence of symptoms, blood cell counts, and an evaluaion of immune system. It is geared to protect uninfected cells, to preserve the immune system, and to slow the progression to AIDS or AIDS-related illness. Recent reports on the numbers of AIDS incidence are less than projected, perhaps due to treatment (Gail et al. [1990]). However, a recent study on the use of AZT and AP in the treatment of patients with symptomatic HIV infection in San Francisco (Lang et al. [1991]) shows surprisingly low number of patients under treatment. Another factor for the lack of comprehensive treatment program for AIDS might be the general perception of AIDS as a disease of outcasts, i.e., homosexuals and drug users. But with the increasing awareness of the general public and the recent findigs about the increasing importance of heterosexual transmission in the spread of the disease one can foresee a more comprehensive treatment program for AIDS patients.

In light of the present situation, on can question what is the effect of the treatment on the population, not at the individual level, but on a population scale; i.e., whether treatment will have an impact on the reduction of prevalence levels and/or the reduction of the incidence rate. Recent studies on the effect of therapy on AIDS incidence include Hethcote et al. [1991] which incorporates therapy in a simulation model of AIDS among gay men in San Francisco, although the results are inconclusive with respect to the effects on HIV transmission rate; and Gail et al. [1990] which points to the possible connection between therapy and a decrease in AIDS incidence due its effect on AIDS incubation and survival time. The question of interest here is to explore the possible consequences of medical treatment of HIV infected persons and the associated behavioral changes on the transmission of the virus to susceptible individuals in a homosexual population.

In Section 2, we propose a model of HIV transmission in a male homosexual population undergoing a treatment program. Treatment is defined to be any combination of medication or other forms of therapy (e.g. 
psychotherapeutic sessions) which may result in a net decrease of the probability of transmission of HIV virus for the individuals undergoing treatment. Hence, in the model we incorporate a (possible) change in sexual behavior once someone is in treatment since change in sexual behavior (use of condoms, avoiding high-risk sexual practices, and so on) is crucial element in the extent of spread of the HIV infection. Studies have shown that the use of condom greatly reduces the probablity of transmission, albeit not completely. Without a cure or a vaccine against AIDS, change in sexual behavior is necessary in the prevention of AIDS. Many studies have shown a significant change in behavior toward safer sexual behavior among gay men, particularly in large urban areas. (See e.g., Becker and Joseph [1988], Stall et al. [1988], Catania et al. [1989], Judson et al. [1989], or 0'Reilly et al. [1989].) On the other hand, reports of unchanged (unsafe) sexual behavior among gay men in low AIDSprevalence areas are also in abundance. (See p. 83, Miller et al. [1990] for a partial list of such reports from 1988 to 1990). In fact, one study on homosexual men in the Netherlands (Griensven et al. [1989]) reported that the seropositive individuals were more likely to have high-risk anal intercourse with their nonsteady partners than seronegative and untested men, although they were more likely to use condoms. Hence, not all individuals can be counted on to change their sexual habits and the picture on the relationship between AIDS and behavioral change is not altogether clear. In most studies on mixing pattern (e.g., Jacquez et al.[1988], Jacquez et al. [1989], Koopman et al. [1989]), the level and pattern of sexual contact are assumed to be constant throughout one's (active) lifetime. Recently, Scalia-Tomba [1991] proposed a model which describes the dynamics of change in sexual behavior from a high activity stage to a low activity stage and vice versa. In this work, by assuming a change in behavior of an individual undergoing treatment, we are assuming essentially that a person with enough sense of responsibility to continue treatment program will reflect it in his sexual practices. We consider only a male homosexual population (no drug users or bisexuals). We also assume distinct probablity of transmission of HIV virus for patients in treatment since, although there is no medical evidence of any drug decreasing the transmission probability, there is strong evidence that use of condom will. 
Section 3 is devoted to qualitative analysis of the model at the disease-free equilibrium with the aim of determining the relative importance of changes in incubation time, probability of transmission, and sex behavior for an HIV infective under treatment. We also explore the relationship between the extensiveness of the treatment program and the prevention of epidemic, if any. Section 4 gives the results on the existence and uniqueness of the endemic equilibrium. Finally we give our conclusions in Section 5.

\section{Model formulation}

Consider a population of homosexual men subdivided into three groups: $S$ (susceptibles), $U$ (the recent infectives not yet in treatment), and I (infectives undergoing treatment). The treatment, as mentioned before, includes any program of medical supervision/therapy of an infective which will have an effect on the transmission of HIV virus.

The model describing the transmission dynamics of HIV virus within the population is then given as follow (the symbol ' ', indicates derivative with respect to time):

$$
\begin{aligned}
& \mathrm{S}^{\prime}(\mathrm{t})=\Lambda-\mathrm{B}(\mathrm{t})-\mu \mathrm{S}+\delta \mathrm{I} \\
& \mathrm{U}^{\prime}(\mathrm{t})=\mathrm{B}(\mathrm{t})-(\mu+\nu) \mathrm{U}-\sigma \mathrm{U} / \mathrm{T} \\
& \mathrm{I}^{\prime}(\mathrm{t})=\sigma \mathrm{U} / \mathrm{T}-\left(\mu+\nu^{\prime}+\delta\right) \mathrm{I}
\end{aligned}
$$

with $T(t)=S(t)+U(t)+I(t)$ being the total population.

Here $\Lambda$ is the constant recruitment rate; $\mu$ is the mortality rate due to causes other than AIDS; $\quad B(t)$ is the force of infection term and $\nu$ and $\nu$ ' are mean death rates due to AIDS of the untreated class and the treatment class, respectively; $\sigma$ is the fixed number of infectives enter into 
treatment per unit time given a population of all untreated infectives; and $\delta$ is the cure rate of the treatment class where one can be cured and sent back to the susceptible class. Although currently $\delta$ is equal to zero.

It should be noted that in an AIDS model with screening proposed by Hsieh [1991], $\sigma$ is the fixed number of individuals screened during each time interval and subsequently, $\sigma \mathrm{U} / \mathrm{T}$ is the proportion of individuals screened to be HIV-positive and removed from the active population per unit time. The implicit assumption in that model is that all individuals tested positive can be successfully removed from activities likely to be of risk to the susceptible class by means of education, changes in behavior, etc. The distinction being made in the present model is that those individuals are not removed, but are taken into a separate treatment class in the active population where possible changes in AIDS-related death rates, probability of HIV transmission, and behavior might occur. This is a more realistic view of the current situation regarding AIDS patients. In fact, one could argue that the number of newly treated infectives should be proportional to the AIDS prevalence in the total population, in which case the treatment is $\sigma / \mathrm{T}$. Another way to look at the treatment term is to view the number of individuals entering into treatment to be proportional to the prevalence of AIDS in the total untreated population since change in behavior might be directly related to the AIDS prevalence (Miller et al. $[1990])$, then $\sigma$ is just the constant of proportionality and the treatment term is $\sigma /(\mathrm{S}+\mathrm{U})$. It will be shown that this change in treatment term does not alter the results of the anlysis in Section 3 and therefore is not important to make such distinction in this article.

The force of infection, $B(t)$, is given by

$B(t)=S(t)\left(\operatorname{cap} \frac{U}{T}+c^{\prime} a^{\prime} p^{\prime} \frac{I}{T}\right)$

where $a$ is the infectivity rate of a susceptible when in contact with an untreated infective, $c$ is the contact rate (the average number of sexual partners per unit time) of a susceptible individual with individuals in class $U$, and $p$ is the probability that an untreated infective engages in sexual activity with a susceptible person given that they have formed a pair; a', c' and p' are the corresponding parameters for the pair formation of a susceptible individual with an infective in the treatment class. 
Hence, $p$ and p' are conditional probabilities with $\mathbf{p}+\mathbf{p}^{\prime}=1$. Moreover, we assume $\mathbf{p}^{\prime}$, the the fraction of contact of susceptibles with the treatment class, is dependent on $\sigma$, i.e.,

$$
\begin{aligned}
\mathbf{p}^{\prime} & =0 & & \text { if } \sigma=0 \\
& =\hat{\mathbf{p}} & & \text { if } \sigma>0 .
\end{aligned}
$$

where $\hat{\mathbf{p}}$ is some fixed fraction. Furthermore, it follows that

$$
\begin{array}{cl}
\mathbf{p} & =1 \quad \text { if } \sigma=0, \\
& =1-\hat{\mathrm{p}} \quad \text { if } \sigma>0 .
\end{array}
$$

The above formulation indicates that without a treatment program $(\sigma=0)$, all contacts with the infectives occur with the untreated class. However with the existence of a treatment class $(\sigma>0)$, there will be infections due to contacts with either infective classes. Based on the assumption that changes in sexual behavior that diminish the transmission rate are undertaken by individuals in the treatment class, we can assume $\hat{\mathbf{p}}<1 / 2$, i.e. the pairing of susceptible individuals with members of the treatment class are no greater than (and in all likelihood less than) those with the untreated infectives. It follows then that $\mathbf{p}$ ' $<$ p. We also assume that the average number of contacts of a susceptible with someone under treatment, c', will be less than or equal to the contacts with an untreated infective due to behavioral change. Futhermore, a' $<$ a since treatment does not increase, and may possibly decrease, the transmission rate. Hence we have

$$
\text { c'a'p' < c a p. }
$$

The net effect of treatment on the transmission of the disease is assumed to be reflected in relation (2.5).

However, the same cannot be said regarding the mean death rates due to AIDS, $\nu$ and $\nu$, although the treatment does not shorten the survival time of the patient (and probably prolongs it). The reason being that while $\nu$ is the mean death rate due to AIDS in the sense that $1 / \nu$ is the mean duration of 
infectiousness (or the average time from infection to full-blown AIDS), $1 / \nu$ ' is the mean duration of time spent by an infective individual under treatment regardless of how long a patient has been infected prior to entering into this class. Therefore, even if we know for certain that, on the average, treatment will retard the progression to full-blown AIDS, $\nu$ ' could still be greater than $\nu$ if the patients entering treatment are heavily concentrated with individuals infected for a long period of time - a likely situation. Thus no assumption can be made concerning the relative size of $\nu$ and $\nu$ '.

\section{Analysis of the model}

Since the disease-free equilibrium of the system $(2.1)-(2.3)$ is $(\Lambda / \mu, 0,0)$, the well-known basic reproductive number, the number of secondary infections caused by an infective among a population of susceptibles in one infectious period, is

$$
\mathrm{R}=\frac{\beta}{\mu+\nu+\sigma \mu / \Lambda}+\frac{\beta^{\prime}}{\mu+\nu^{\prime}+\delta} \frac{\sigma \mu}{\Lambda(\mu+\nu)+\sigma \mu}
$$

where we have labeled $\beta=$ cap and $\beta^{\prime}=\mathrm{c}^{\prime} \mathrm{a}^{\prime} \mathrm{p}$ '. In figure 1 it is shown the dependence of $\mathrm{R}$ on $\beta^{\prime}$ and $\sigma$.

In epidemiological studies, the basic reporductive number is closely related to the outcome of an epidemic by the simple criterion that $R>1$ implies persistence of epidemic while $R<1$ means the disease will die out. In the following portion of this article, we will look more closely at this condition but before going into more detailed analysis we explore the epidemiology of the early stages of the disease according to our model.

(FIGURE 1 ABOUT HERE)

At the begining of the epidemic we can assume that all members of the population are susceptible which translates into $S \cong T$ and we can take this quantity to be roughly constant by neglecting recruitment and mortality (Anderson and May, 1991). At this early stage there are no infectious individuals undergoing treatment and thus we obtain the following equation for $U$ :

$$
\mathrm{U}^{\prime}(\mathrm{t})=(\beta-\nu-\sigma / \mathrm{T}) \mathrm{U}(\mathrm{t})
$$


where $\sigma / \mathrm{T}$ is assumed constant. The solution to this equation is

$$
\mathrm{U}(\mathrm{t})=\mathrm{U} \exp [(\beta-\nu-\sigma / \mathrm{T}) \mathrm{t}]
$$

which renders the initial doubling time for the epidemic ( the time required for doubling the number of individuals initially infected with the virus)

$$
\theta=0.7 /(\beta-\nu-\sigma / \mathrm{T})
$$

the expression is similar to that obtained by Anderson and May (1991) differing only in the iclusion of $\sigma / \mathrm{T}$ which lengthens the doubling time.

Notice that at the beginning of the infection the number of cases rises exponentially with growth rate equal to

$$
\beta-\nu-\sigma / \mathrm{T}
$$

The above relation sets also an upper limit for the treatment rate $\sigma / \mathrm{T}$ which is

$$
\sigma / \mathrm{T}<\beta-\nu
$$

indicating that to stop the spread of the epidemic in its early stages treatment rate has to be equal to the net recruitment rate of newly infected persons situation that is unlikely given the difficulties inherent in the determination of the incidence rate of the disease.

When $\sigma=0$, i.e. no one in the population is being treated, we have $\mathrm{I}=0$. In this case the system (2.1)-(2.3) simplifies to

$$
\begin{aligned}
& \mathrm{S}^{\prime}(\mathrm{t})=\Lambda-\beta \mathrm{S} \frac{\mathrm{U}}{\mathrm{S}+\mathrm{U}} \\
& \mathrm{U}^{\prime}(\mathrm{t})=\beta \mathrm{S} \frac{\mathrm{U}}{\mathrm{S}+\mathrm{U}}-(\mu+\nu) \mathrm{U}
\end{aligned}
$$


which are exactly Equations (2.5)-(2.6) in Hsieh [1991]. Therefore we have the following result:

Proposition 1. Let $R=\beta /(\mu+\nu)$. If $R<1$, the disease-free equilibrium $(\Lambda / \mu, 0)$ is the unique equilibrium for system (2.1)-(2.3) and is globally asymptotically stable. If $R>1$, there exists a unique endemic equilibrium ( $\tilde{\mathrm{S}}, \tilde{\mathrm{U}})$ which is asymptotically stable for all initial populations except at the disease-free equilibrium.

For proof of this proposition, see Hsieh [1991].

Our concern then is to know whether a positive value of $\sigma$ will prevent the convergence of populations toward an endemic population when $\mathrm{R}>1$. Recall that $\sigma>0$ implies, for our model, that $\beta>\beta^{\prime}$ or, in other words, that $\sigma>0$ comes together with a reduction of risky sexual behaviors. The Jacobian matrix of system (2.1)-(2.3)

At the disease-free equilibrium $(\Lambda / \mu, 0,0)$ the Jacobian matrix becomes 
The eigenvalues of the matrix are $-\mu$ and the roots of the characteristic equation

$$
\lambda^{2}+\left(\frac{\sigma \mu}{\Lambda}+2 \mu+\nu+\nu^{\prime}-\beta\right) \lambda+\left(\mu+\nu^{\prime}\right)\left(\frac{\sigma \mu}{\Lambda}+\mu+\nu-\beta\right)-\beta^{\prime} \frac{\sigma \mu}{\Lambda}=0 .
$$

Performing some elementary computations and using the Routh-Hurwitz criterion, we obtain the follow result on the stability of the matrix:

Proposition 2. Given $\sigma>0$ and $\beta>\mu+\nu$ we have

(a), The matrix is unstable if $\beta^{\prime}>\mu+\nu^{\prime}+\delta$.

(b). When $\beta^{\prime}<\mu+\nu^{\prime}+\delta$, then the matrix is unstable if $\sigma<\tilde{\sigma}$, and it is stable if $\tilde{\sigma}<\sigma ;$ where

$$
\tilde{\sigma}=\frac{\Lambda}{\mu} \frac{\beta-(\mu+\nu)}{\mu+\nu^{\prime}+\delta-\beta^{\prime}}(\mu+\nu+\delta)
$$

Proposition 2 gives us the local stability result of system (2.1)-(2.3) at the disease-free equilibrium. First we make the observation that $\tilde{\sigma}>\sigma$ is equivalent to $\mathrm{R}>1$. Hence we can state the local stability property of the system (2.1)-(2.3) at disease-free equilibrium can be stated in terms of the basic reproduction number:

Proposition 3. Given $\beta>\mu+\nu$ and $\delta>0$. If $\beta^{\prime}>\mu+\nu^{\prime}+\delta$, then $\mathrm{R}>1$ for all $\sigma>0$ and the disease-free equilibrium is unstable. If $\beta^{\prime}\left\langle\mu+\nu^{\prime}+\delta\right.$ then the disease-free equilibrium is unstable if $\mathrm{R}>1(\tilde{\sigma}>\sigma)$, and locally asymptotically stable if $\mathrm{R} \leq 1(\tilde{\sigma} \leq \sigma)$. 
Note that if the treatment term is changed to $\sigma /(\mathrm{S}+\mathrm{U}), \mathrm{R}$ remains the same. The Jacobian matrix $J$ will be different but, at the disease-free equilibrium, $J$ will be the same as in (3.4) and hence Propositions 1, 2, and 3 follow similarly. Therefore having the treatment term proportional to the ratio of the untreated to the total population or the total untreated population does not alter our results.

It is interesting to note that when $\beta^{\prime}=c^{\prime} a^{\prime} p^{\prime}$ is too large, the disease will persist no matter how comprehensive the treatment program is. However, if $\beta^{\prime}\left\langle\mu+\nu^{\prime}+\delta\right.$, the convergence of the population will depend on whether the treatment program is comprehensive enough compared to the threshold value for the size of treatment program.

4. Characterization of the endemic equilibrium

To explore the behavior of the system when the disease-free equilibrium is unstable, we make the assumption that $\delta=0$ and $\nu=\nu^{\prime}=\hat{\nu}$. To that end define the new variables

$$
\mathrm{t}^{\prime}=\mu \mathrm{t}, \quad \mathrm{b}=\beta / \mu, \quad \mathrm{b}^{\prime}=\beta^{\prime} / \mu, \quad \theta=1+\nu / \mu, \quad \sigma^{\prime}=\frac{\sigma}{\Lambda}
$$

and

$$
\tilde{\mathrm{S}}=\frac{\mu \mathrm{S}}{\Lambda}, \quad \tilde{\mathrm{U}}=\frac{\mu \mathrm{U}}{\Lambda}, \quad \tilde{\mathrm{I}}=\frac{\mu \mathrm{I}}{\Lambda}
$$

With these new variables, system (2.1)-(2.3) is given by

$$
\frac{d}{d t^{\prime}} \tilde{S}=1-\tilde{B}\left(t^{\prime}\right) \tilde{S}-\tilde{S}
$$




$$
\begin{aligned}
& \frac{\mathrm{d}}{\mathrm{dt}^{\prime}} \tilde{\mathrm{U}}=\tilde{\mathrm{B}}\left(\mathrm{t}^{\prime}\right) \tilde{\mathrm{S}}-\theta \tilde{\mathrm{U}}-\sigma \frac{\tilde{\mathrm{U}}}{\tilde{\mathrm{T}}}, \\
& \frac{\mathrm{d}}{\mathrm{dt}^{\prime}} \tilde{\mathrm{I}}=\sigma^{\prime} \frac{\tilde{\mathrm{U}}}{\tilde{\mathrm{T}}}-\theta \tilde{\mathrm{I}},
\end{aligned}
$$

with $\tilde{\mathrm{T}}\left(\mathrm{t}^{\prime}\right)=\tilde{\mathrm{S}}\left(\mathrm{t}^{\prime}\right)+\tilde{U}\left(\mathrm{t}^{\prime}\right)+\tilde{\mathrm{I}}\left(\mathrm{t}^{\prime}\right)$ and $\tilde{\mathrm{B}}=\mathrm{b} \frac{\tilde{\mathrm{U}}}{\tilde{\mathrm{T}}}+\mathrm{b}^{\prime} \frac{\tilde{\mathrm{I}}}{\tilde{\mathrm{T}}}$

Setting the RHS of (4.1)-(4.3) equal to zero we find the expression for the coordinates of the endemic equilibria

$$
\hat{\mathrm{S}}=\frac{1}{1+\hat{\mathrm{B}}}, \quad \hat{\mathrm{U}}=\frac{\hat{\mathrm{B}} \hat{\mathrm{S}}}{\theta+\sigma^{\prime} / \hat{\mathrm{T}}^{\prime}}, \quad \hat{\mathrm{I}}=\frac{\sigma^{\prime}}{\theta+\hat{\mathrm{T}}^{\circ}}
$$

Substituting (4.4) into the expression for $\hat{\mathrm{B}}$ and rearranging terms we obtain

$$
\hat{\mathrm{B}}=\frac{\mathrm{b}+\frac{\mathrm{b}^{\prime}}{\theta \hat{\mathrm{T}}^{2}} \sigma^{\prime}}{\hat{\mathrm{T}} \theta+\sigma^{\prime}}-1
$$

Since $\mu<\nu$ it is not possible to find an explicit expression for $\hat{\mathrm{T}}$. However, some information about the nature of the equilibrium point can still be obtained. Adding together the expressions in (4.4) one obtains, for $\mathrm{T}>0$

$$
\hat{\mathrm{T}}=\frac{1}{1+\hat{\mathrm{B}}}\left(1+\frac{\hat{\mathrm{B}}}{\hat{\mathrm{T}} \theta+\sigma^{\prime}}+\frac{\sigma^{\prime} \hat{\mathrm{B}}}{\theta \hat{\mathrm{T}}^{2}\left(\hat{\mathrm{T}} \theta+\sigma^{\prime}\right)}\right)
$$

which together with (4.6) gives a system of non-linear algebraic equations whose solutions correspond to the possible equilibria of system (4.1). Substituting (4.5) into (4.6) we have (to keep the notation simple enough we drop the " ' from the state variables below) 
$\mathrm{T}=\frac{\theta^{4} \mathrm{~T}^{6}+\theta^{3}\left(2 \sigma^{\prime}-1\right) \mathrm{T}^{5}+\theta^{2}\left(\mathrm{~b}-\sigma^{\prime}\left(1-\sigma^{\prime}\right)\right) \mathrm{T}^{4}-\sigma^{\prime} \theta^{2} \mathrm{~T}^{3}+\sigma^{\prime} \theta\left(\mathrm{b}+\sigma^{\prime}(\mathrm{b}-1)\right) \mathrm{T}^{2}+\mathrm{b}^{\prime} \sigma^{\prime 2}}{\mathrm{~b} \theta^{3} \mathrm{~T}^{5}+\mathrm{b} \sigma \theta^{2} \mathrm{~T}^{4}+\mathrm{b}^{\prime} \sigma^{\prime} \theta^{2} \mathrm{~T}^{3}+\mathrm{T}^{2} \mathrm{~b}^{\prime} \sigma^{2} \theta}$.

Denoting by $\mathrm{F}(\mathrm{T})$ the RHS of (4.7), we prove now the existence of a fixed point of $\mathrm{F}$. Notice first that all coefficients except the one corresponding to $\mathrm{T}^{3}$ in the numerator of $\mathrm{F}$ are positive since $\sigma^{\prime}>>1$ and b $>1$. Secondly we look for fixed points only for values of $T$ in $[0,1]$. The reason being that at the disease-free equilibrium we have all the population being susceptible, i.e., $\mathrm{T}=\mathrm{S}=1$ (remember that we are using the rescaled variables defined at the beginnig of the section). We thus expect that when the virus spreads the value of $S$ at equilibrium will actually go down below 1 and that the total population will in fact satisfy $T<1$. Also note that as $T$ goes to zero $F(T)$ tends $+\infty$ and that for $0<$ $\mathrm{T}<1$ it is always positive. Evaluating $\mathrm{F}$ at $\mathrm{T}=1$ we obtain

$$
F(1)=\frac{b^{\prime} \sigma^{\prime}+\theta\left(b-\sigma^{\prime}\right)+\theta^{2}\left(\sigma^{\prime}-1\right)+\theta^{3}}{\theta\left(b^{\prime} \sigma+b \theta\right)}
$$

$\mathrm{F}(1)$ is positive since $\left|\mathrm{b}-\sigma^{\prime}\right|<\left|\sigma^{\prime}-1\right|$ and $\theta>1$. Moreover $\mathrm{F}(1)<1$ since this is equivalent to require that

$$
\theta \sigma^{\prime}+\theta^{2}<b^{\prime} \sigma^{\prime}+\mathrm{b} \theta
$$

and, if the disease-free equilibrium is unstable (i.e., the disease is spreading) we have $\theta<\mathrm{b}$ which in terms of the original parameters can be written as $\beta>\mu+\nu$. This condition guaranties (4.8). (see figure 3).

(FIGURE 3 ABOUT HERE ILLUSTRATING 4.8)

We conclude the preceeding discussion with 
Proposition 4. If condition (4.8) holds, there exists at least one endemic equilibrium point for system (4.1)-(4.3) (and hence the same hold for system (2.1)-(2.3)).

Proof: The condition in the hypothesis implies that there is a $\tilde{\mathrm{T}}$ such that $\mathrm{F}(\tilde{\mathrm{T}})=\tilde{\mathrm{T}}$ with $0<\tilde{\mathrm{T}}<1$.

Notice that for $\epsilon$ small

$$
\mathrm{F}(\epsilon) \sim \frac{1}{\epsilon^{2} \theta}=\mathrm{O}\left(\frac{1}{\epsilon^{2}}\right)
$$

and, by Decartes' rule of signs $\mathrm{F}$ has no poles in $(0,1]$. Also, by Decartes' rule of signs the numerator of $F(T)$ has no positive zeroes since the coefficients of the polynomial change sign only twice. Thus the graph of $F$ always remains above the horizontal axis. We now show that for certain (feasible) values of $\theta, b, b^{\prime}$ and $\sigma^{\prime}, F(T)$ is monotonically decreasing in $(0,1]$. Notice that the changes of sign of the derivative with respect to $T$ of $F$ (denoted $F^{\prime}(T)$ ) are determined by the changes of sign of the derivative of the numerator of the RHS of (4.7). Define $p(T)$ as the numerator of $F(T)$ divided by $\theta^{4}$ (so as to obtain a monic polynomial). Then

$$
\frac{\mathrm{dp}}{\mathrm{dT}}=6 \mathrm{~T}^{5}+5 \theta\left(2 \sigma^{\prime}-1\right) \mathrm{T}^{4}+4 \theta^{-2}\left(\mathrm{~b}-\sigma^{\prime}\left(1-\sigma^{\prime}\right)\right) \mathrm{T}^{3}-3 \sigma^{\prime} \theta^{-2} \mathrm{~T}^{2}+2 \sigma^{\prime} \theta^{-3}\left(\mathrm{~b}+\sigma^{\prime}(\mathrm{b}-1)\right) \mathrm{T} .
$$

We apply again Decarte's rule to $\mathrm{p}^{\prime}(\mathrm{T}) / \mathrm{T}$ and obtain that it has no real positive zeroes for $\mathrm{T}>\mathbf{0}$

We use now known results from Henrici (1974, pp: 450-453). Define

$$
b_{m}=\frac{1}{m !} p^{(m)}\left(T_{0}\right), \quad m=0,1, \ldots, n \quad\left(b_{n}=1\right)
$$

where $p^{(m)}$ denotes the mth derivative of $p$ evaluated at $T_{0}$.

Then the polynomial

$$
\rho^{\mathrm{n}}+\left|\mathrm{b}_{\mathrm{n}-1}\right| \rho^{\mathrm{n}-1}+\ldots+\left|\mathrm{b}_{2}\right| \rho^{2}+\left|\mathrm{b}_{1}\right| \rho-\left|\mathrm{b}_{0}\right|
$$

has exactly one positive solution. Let this solution be $\hat{\rho}$. The following theorem holds.

Theorem 1. (Henrici, 1974 p. 452) No zero of $\mathrm{p}$ is contained in the open disk $\left|\mathrm{T}-\mathrm{T}_{0}\right|<\hat{\rho}$. 
We have the following proposition regarding the uniqueness of the endemic equilibrium of system (4.1)(4.3).

Proposition 5. There exist positive numbers $\theta_{0}, b_{0}, b^{\prime}{ }_{0}$, and $\sigma_{0}$ such that for $\left|\theta-\theta_{0}\right|,\left|b-b_{0}\right|$, $\left|b^{\prime}-b^{\prime}{ }_{0}\right|$ and $\left|\sigma^{\prime}-\sigma_{0}\right|$ small enough. Then there exist a unique solution to equation (4.7) for $T$ in the interval $[\epsilon, 1]$ where $\epsilon$ is arbitrarily small.

Proof: As pointed out before, changes in sign of $\mathrm{F}^{\prime}$ are determined by the changes in sign of (4.10). For fixed $\epsilon>0$ let $\left\{\mathrm{T}_{\mathrm{j}}\right\}, \mathrm{j}=1, \ldots, \mathrm{k}$, denote a partition of the interval $[\epsilon, 1]$. For each $\mathrm{T}_{\mathrm{j}}$ we apply now Theorem 1. Since $[\epsilon, 1]$ is compact and $p^{\prime}(T) / T$ does not have positive real zeroes, there exists a $k_{*}$ for which the set $\left\{\left|\mathrm{T}-\mathrm{T}_{\mathrm{j}}\right|\right\}, \mathrm{j}=1, \ldots, \mathrm{k}_{*}$ generated by the repeated use of Theorem 1 is a finite open cover. It follows that (4.7) and hence $F^{\prime}$ do not change sign in $[\epsilon, 1]$. This implies that $F$ is monotone in $[\epsilon, 1]$ rendering the uniqueness of the endemic equilibrium point in this interval. The existence of $\theta_{0}, b_{0}, b^{\prime}{ }_{0}$, and $\sigma^{\prime}{ }_{0}$ is dictated by biological feasibility. In figure 4 we illustrate the existence of the fixed point for one set of parameter values Q.E.D.

It is important to note that $\epsilon$ is a function of the parameter values $\theta_{0}, b_{0}, b_{0}^{\prime}$, and $\sigma_{0}^{\prime}$.

We discuss now some of the properties of the endemic equilibrium of system (4.1)-(4.3). Define as

$$
\phi\left(\sigma^{\prime}, \hat{\mathrm{T}}\right)=\frac{\mathrm{b}+\frac{\mathrm{b}^{\prime}}{\theta \hat{\mathrm{T}}^{2}} \sigma^{\prime}}{\hat{\mathrm{T}} \theta+\sigma^{\prime}}
$$

the first term on the RHS of (4.5). Using (4.4) and (4.5) we have

$$
\hat{\mathrm{S}}=\frac{1}{\phi}, \quad \hat{\mathrm{U}}=\frac{\phi-1}{\phi\left(\hat{\mathrm{T}} \theta+\sigma^{\prime}\right)}, \quad \hat{\mathrm{I}}=\frac{\sigma^{\prime}(\phi-1)}{\hat{\mathrm{T}}^{2} \theta^{2} \phi} .
$$

The magnitude of the populations at equilibrium depends on the properties of $\phi$. Of interest in this 
work is the nature of the relationship between $\phi$ and $\sigma^{\prime}$ and $\mathrm{T}$, the total population. In figure \# we show graphically the nature of this relationship. Specifically we have

$$
\frac{\partial \phi}{\partial \sigma^{\prime}}=\frac{\mathrm{Tb}^{\prime} \theta-\mathrm{b}(\theta+\mathrm{T})}{(\mathrm{T}+\theta)\left(\sigma^{\prime}+\mathrm{T} \theta\right)^{2}}<0
$$

if and only if $\theta b^{\prime}<b$. This relation holds only for very small values of $b^{\prime}$ (see figure 5).

(FIGURE 4 ILLUSTRATING $\phi$ AS A FUNCTION OF $\sigma$ and T ABOUT HERE)

(FIGURE 5 ILLUSTRATING WHEN THE LAST RELATION HOLDS ABOUT HERE)

This means that in order for treatment to be effective bringing down the incidence rate of HIV, it is necessary to enforce behaviors that effectivelly reduce the transmission rate b' otherwise, no matter how large $\sigma^{\prime}$ is the incidence rate will be always positive.

Note that $\phi$ depends on the size of the total population. In this case it can be verified that

$$
\frac{\partial \phi}{\partial \mathrm{T}}<0
$$

for all feasible parameter values due to the fact that in a larger population the contacts of infective individuals in either class is diluted among all teh members (this is a consequence of assuming an homogeneous population and proportional mixing for the probabilities $p$ and p' in (2.1)-(2.3) ).

To wrap up the discussion and analysis of this section, we study now a particular case of (2.1)-(2.1) for which the coordinates of the endemic equilibrium point can be explicitly computed. In this case we take $\nu=\nu^{\prime}=0$ neglecting disease induced mortality. This assumption stresses the action of $\beta, \beta^{\prime}$ and $\sigma$ on the spread of the disease. Thus, from (2.1)-(2.3) we have 
Thus, from (2.1)-(2.3) we have

$$
\mathrm{T}^{\prime}(\mathrm{t})=\boldsymbol{\Lambda}-\boldsymbol{\mu} \mathrm{T}
$$

and, at equilibrium,

$$
\hat{\mathrm{T}}=\frac{\Lambda}{\mu}
$$

Setting the RHS of (2.1)-(2.3) equal to zero we obtain

$$
\hat{\mathrm{S}}=\frac{\Lambda}{\hat{\mathrm{B}}+\mu}, \quad \hat{\mathrm{U}}=\frac{\hat{\mathrm{B}} \hat{\mathrm{S}}}{\mu+\sigma / \hat{\mathrm{T}}}, \quad \hat{\mathrm{I}}=\frac{\sigma \hat{\mathrm{U}}}{\hat{\mathrm{T}}}
$$

where

$$
\hat{\mathrm{B}}=\beta \frac{\hat{\mathrm{U}}}{\hat{\mathrm{T}}}+\beta^{\prime} \frac{\hat{\mathrm{I}}}{\hat{\mathrm{T}}}
$$

is the expression for the force of infection $B(t)$ at equilibrium. Substituting (4.1) and (4.2) into (4.3) we obtain that

$$
\hat{\mathbf{B}}=\mu(\phi-1)
$$

where

$$
\phi=\frac{\beta+\beta^{\prime} \frac{\sigma}{\Lambda}}{\mu\left(1+\frac{\sigma}{\Lambda}\right)}
$$

With this expression for the force of infection we obtain from (4.2)

$$
\hat{\mathrm{S}}=\frac{\Lambda}{\mu \phi}, \quad \hat{\mathrm{U}}=\frac{\Lambda(\phi-1)}{\phi \mu\left(1-\frac{\sigma}{\Lambda}\right)}, \quad \hat{\mathrm{I}}=\frac{\sigma \Lambda(\phi-1)}{\phi \mu\left(1-\frac{\sigma}{\Lambda}\right)}
$$


The point $(\hat{\mathrm{S}}, \hat{\mathrm{U}}, \hat{\mathrm{I}})$ given by (4.5) is the unique endemic equilibrium point for system (2.1)-(2.3) when we assume no cure rate $(\delta=0)$ and $\nu=\nu$. Notice that $\phi=1$ gives the disease-free equilibrium of the model. Also since

$$
\frac{\mathrm{d} \phi}{\mathrm{d} \sigma}=\frac{\Lambda}{\mu(\Lambda+\sigma)^{2}}\left(\beta^{\prime}-\beta\right) \leq 0
$$

we see that tretment can reduce the prevalence level of the disease in both types of infective individuals provided there are associated behavioral changes that insure $\beta^{\prime}<\beta$. Otherwise, no matter how large $\sigma$ is, prevalence levels will always be away from zero. If we solve the equation $\phi=1$ for $\sigma$ we obtain

$$
\sigma=\frac{\Lambda(\mu-\beta)}{\beta^{\prime}-\mu}
$$

as the value for $\sigma$ that guaranties the prevalence levels to be zero. The value of $\sigma$ given by (4.7) is positive (biologically feasible) only if two conditions are satisfied. One of them is

$$
\beta^{\prime}<\mu<\beta
$$

This indicates that $\beta$ ' has to be substantially small in order for treatment to succeed. For example, in a population where the life expectancy at birth is of 70 years, $\beta^{\prime}$ has to be less than $1 / 70$ in order for a treatment program to bring down the prevalence level of HIV infection. The other is that $\sigma$ can not be arbitrarily large since the number of infective individuals going into treatment can be at most he total number of untreated infectives existing at any time. Thus, even when (4.8) holds $\sigma$ may still not be feasible and (4.7) has no solution. We finally point out that the discussion presented in this section up to this point is valid only when the disease has reached the endemic equilibrium given by (4.5). In this 
contest, if condition (4.8) does not hold there is no treatment rate that results in $\phi=1$. If $\mu<\beta^{\prime}<\beta$ the transmission probabilities are so high that the incidence rate $B$ is always positive and large producing high prevalence levels even if the populaton is under a treatment program. If $\beta^{\prime}<\beta<\mu$, the endemic equilibrium does not exists.

We now proceed with the stability analysis of the equilibrium point (4.5). Equation (3.1) can be rewritten as

$$
\mathrm{R}\left(\beta^{\prime}, \sigma\right)=\frac{1}{\mu+\nu+\sigma \mu / \Lambda}\left(\beta+\beta^{\prime} \frac{\sigma \mu}{(\mu+\nu) \Lambda}\right)
$$

Since the parameters all are positive, $\mathrm{R}$ is a continuous function in the variables $\beta^{\prime}$ and $\sigma$. When $\beta=\beta^{\prime}, R\left(\beta^{\prime}, \sigma\right)>1$ and the disease-free equilibrium is unstable. Furthermore, in this case there exists a unique endemic equilibrium $(\hat{\mathrm{S}}, \hat{\mathrm{U}}, \hat{\mathrm{I}})$ given by (4.5).

Using a continuity argument, together with Proposition 3, we have the main result for the system (2.1)-(2.3) in a small neighborhood of $|\nu-\nu|$ and $\delta$.

Proposition 6. Given $\left|\nu-\nu^{\prime}\right|$ small, $\delta$ small and positive, and $\beta>\mu+\nu$, if $\beta^{\prime} \leq \mu+\nu+\delta$, then the disease-free equilibrium for system (2.1)-(2.3) is unstable and there exists a unique endemic equilibrium. If, however, $\beta^{\prime}<\mu+\nu+\delta$, then the disease-free equilibrium is stable provided $\sigma \geq \hat{\sigma}$ and there is no endemic equilibrium. If $\sigma<\hat{\sigma}$ then the disease-free equilibrium is unstable and there exists a unique endemic equilibrium point.

We now prove a stability result for the endemic equilibrium point of system (4.1)-(4.3). To this end let

$$
\Phi(\mathrm{t})=\frac{1}{2}(\hat{\phi}-\phi(\mathrm{t}))^{2}
$$

where $\phi(t)$ is given by (4.11) and $\hat{\phi}$ is $\phi$ evaluated at the endemic equilibrium point (4.12). Thus $\Phi$ evaluated at the endemic equilibrum is zero and positive for all $\mathrm{T}>0$. Moreover, viewing $\phi$ as a function of $\mathrm{T}$ we have 


$$
\frac{\partial \phi}{\partial \mathrm{T}}<0
$$

for all $\mathrm{T}>0$. Thus

$$
\frac{\mathrm{d} \Phi}{\mathrm{dt}}=-(\hat{\phi}-\phi(\mathrm{t})) \frac{\partial \phi}{\partial \mathrm{T}}(\Lambda-(\mathrm{S}+\theta(\mathrm{U}+\mathrm{I}))
$$

Theorem 2. Assume $\phi>1$. Then the unique endemic equilibrium point of system (4.1)-(4.3) is asymptotically stable.

Proof: Since $\phi>1$, the endemic equilibrium point exists and it is unique. We prove now that $\Phi$ is a Lyapunov function of system (4.1)-(4.3). It is positive definite for all $\mathrm{T}>0$ and $\Phi(\hat{\phi})=0$. Also note that the RHS of (4.22) can be rewritten as

$$
-\frac{\partial \phi}{\partial \mathrm{T}}(\mathrm{a}-\mathrm{b})(\hat{\phi}-\phi)
$$

where $\mathrm{a}=\Lambda$ and $\mathrm{b}=\mathrm{S}+\theta(\mathrm{U}+\mathrm{I})$. Thus if the total population sligthly increases we have $\mathrm{a}>\mathrm{b}$ and since $\phi$ is a decreasing function of $\mathrm{T}$ this implies $\frac{\mathrm{d} \Phi}{\mathrm{dt}}<0$. Analogously, if $\mathrm{T}$ decreases we have $\mathrm{a}<\mathrm{b}$ implying $\phi>\hat{\phi}$ rendering $\frac{\mathrm{d} \Phi}{\mathrm{dt}}<0$. It follows that the endemic equilibrium point is globally asymptotically stable. QED.

5. Conclusions. The conclusions of this model indicate that whenever the incidence rate is positive there will be a asimptotically stable endemic equilibrium point no matter the level of treatment to which the population of infective individuals is being subjected to. However, results indicate that even if treatment cannot be effective to eradicate the disease, it can bring down the prevalence. Moreover, the only way in which treatment can have a significant impact either in eradicating the disease or lowering its prevalence is, one, to reach a high proportion of the infective population and two, to greatly alter the risky sexual behavior of the total population. In figures 6 and 7 we illustrate the endemicity levels of HIV whenever one of the two conditions just given is not fullfilled. In both cases the prevalence of the disease is relativelly high.

(FIGURES 6 AND 7 ABOUT HERE)

The Lyapunov function used to prove the global asymptotic stabilty of the endemic equilibrium was 
derived from the observation that the endemic level that any disease can achieve is the result of two interacting factors. One is the incidence rate per se (the number of new cases per unit time), and the other is the number of individuals (regardles of their serological status) that are exposed or re-exposed to the infectious agent per unit time (in this case HIV). In fact, (4.11) shows that in a homogeneous population under proportional mixing the incidence rate of the disease is really a function of the total population. These considerations led us to choose (4.22) as our 'energy' function.

Acknowledgements. This work was possible thanks to the partial financial support to JXVH from funds from the Dean and the Office of Sponsored programs of the College of Agriculture and the Mathematics Science Institute at Cornell University and by NSF grant DMS- 8906580 to Carlos Castillo-Chavez.

\section{REFERENCES}

Anderson, R.M and R.M.May Infectious diseases of Humans. Oxford University Press. Oxford 1991

Becker, M. H., and Joseph, J. G. AIDS and behavioral change to reduce risk: A review. Amer j Public Health 1988, 78:394-410.

Broder, S. and Fauci, A. S. Progress in drug therapies for HIV infection, Public Health Report 103 (May-June 1988):224-28.

Dournon, E., Rozenbaum, W., Michon, C. et al. Effects of zidovudine in 265 consecutive patients with AIDS or AIDS-related complex. Lancet 1988, 2:1297-302.

Fischl, M. A., Richamn, D. D., Causey, D. M. et al. Prolonged zidovudine therapy in patients with AIDS and advanced AIDS-related complex. JAMA 1989, 44:409-11.

Fischl, M.A., Richman D.D., Grieco, M.H., and The AZT Collaborative Working Group. The efficacy of Azidothymidine (AZT) in the treatment of patients with AIDS and AIDS-related complex. A double-blind, placebo-controlled trial. N Engl J Med. 1987, 317:185-91.

Gail, M. H., Rosenberg, P. S., and Goedert, J. J. Therapy may explain recent deficits in AIDS incidence, J Acq Immun Defic Syndr 1990, 3:296-306.

Griensven, G. J. P., de Vroome, E. M. M., Tirlman, R. A. P., Goudsmit, J., de Wolf, F., der Noordaa, 
J., and Courinho, R. A. Effect of human immunodeficiency virus (HIV) antibody knowledge on highrisk sexual behavior with steady and nonsteady sexual partners among homosexual men. Amer J. Epidemiology 1989, 129(3):596-603.

Henrici, P. Applied and Computational Complex Analysis V. I. John Wiley and Sons, New York, 1974) Hethcote, H. W., Van Ark, J. W., and Karon, J. M. A simulation model of AIDS in San Francisco: II. Simulations, therapy, and sensitivity analysis. Math Biosci (to appear).

Hsieh, Y.-H. Modelling the effect of screening in HIV transmission dynamics. Proceedings of International Conference on Differential Equations and its Applications to Mathematical Biology, Claremont, 1990. Lec. Notes in Biomathematics, (p. ?) Springer-Verlag, 1991.

Miller, H. G., Turner, C. F., and Moses, L. E., Ed. AIDS, The Second Decade. National Research Council. 1990. National Academy Press, Washington, D.C.

Lang, W., Osmond, D., Samual, M., Moss, A., Schrager, L., and Winkelstein, Jr., W. Population-based estimates of zidovudine and aerosol pentamidine use in San Francisco: 1987-1989. J Acq Immun Defic Syndr 1991, 4:713-6.

Masur et al.: and Center for Disease Control, Kaposi's Sarcoma and Pneumoncystis Pneumonia among homosexual men - New York City and California. Morbidity and Mortality Weekly report 30 (3 July 1981):305-8.

Phillips, N. C., Skamene, E., and Tsoukas, C. Liposomal encapsulation of 3'-Azido-3'-deoxythymidine (AZT) results in decresed bone marrow toxicity and enhanced activity against murine AIDS-induced immunosuppression. J Acq Immun Defic Syndr 1991, 4:959-66.

Richman, d. d., Fischl, A. M. Grieco, M. H. et al. The toxicity of azidothymidine (AZT) in the treatment of patients with AIDS and AIDS-related complex. A double-blind, placebo-controlled trial. $\mathrm{N}$ Engl J Med 1987, 317:192-7.

Scalia-Tomba, G. The effect of structural behaviour change on the spread of HIV in a one-sex population. Math Biosci (to appear). 